\title{
Relationship between plasma sialic acid and fibrinogen concentration and incident micro- and macrovascular complications in type 1 diabetes. The EURODIAB Prospective Complications Study (PCS)
}

\author{
S. S. Soedamah-Muthu • N. Chaturvedi • J. C. Pickup • \\ J. H. Fuller • and the EURODIAB Prospective \\ Complications Study Group
}

Received: 5 June 2007 / Accepted: 22 November 2007 / Published online: 9 January 2008

(C) The Author(s) 2007

\begin{abstract}
Aims/hypothesis Type 1 diabetes is associated with an increased risk of vascular complications. This increased risk could be explained by sialic acid and/or fibrinogen. It is also not clear what explains the abolition of sex-related differences affecting risk of CHD in the presence of type 1 diabetes. Therefore, we examined whether fibrinogen and sialic acid are related to incident micro- and macrovascular complications in patients with type 1 diabetes.
\end{abstract}

Electronic supplementary material The online version of this article (doi:10.1007/s00125-007-0905-8) contains supplementary material and details of all EURODIAB Prospective Complications Study investigators and staff, and is available to authorised users.

S. S. Soedamah-Muthu $(\bowtie)$

Julius Center for Health Sciences and Primary Care,

University Medical Centre Utrecht,

Heidelberglaan 100, Huispostnr str. 6.131, PO Box 85500,

3508 GA, Utrecht, The Netherlands

e-mail: s.s.soedamah-muthu@umcutrecht.nl

S. S. Soedamah-Muthu $\cdot$ J. H. Fuller

Department of Epidemiology and Public Health,

Royal Free and University College London Medical School,

London, UK

N. Chaturvedi

National Heart and Lung Institute, Imperial College London,

London, UK

J. C. Pickup

Metabolic Unit, King's College London School of Medicine,

Guy's Hospital,

London, UK
Methods A subset $(n=2329)$ of the EURODIAB Prospective Complications Study was analysed. Sialic acid and fibrinogen concentrations were measured at baseline. The main outcomes after 7 years were development of albuminuria, retinopathy, neuropathy and CHD.

Results Univariable and multivariable models using Cox proportional survival analyses showed that an SD unit increase in sialic acid and fibrinogen levels was significantly associated with CHD in men only. Adjusted standardised hazard ratios (sHRs) were 1.50 (95\% CI 1.05-2.15) and 1.40 (95\% CI 1.06-1.86) for sialic acid and fibrinogen, respectively. Initial associations between (1) sialic acid and incident retinopathy [standardised odds ratio (sOR) men $1.68,95 \% \mathrm{CI}$ 1.10-2.57], (2) fibrinogen and retinopathy (sOR women $1.37,95 \%$ CI 1.06-1.78) and (3) sialic acid and neuropathy (sOR men 1.37, 95\% CI 1.06-1.77) were shown, but became non-significant in multivariable models.

Conclusions/interpretation Sialic acid and fibrinogen are strong predictors of CHD in men with type 1 diabetes, beyond the effect of established risk factors. The associations found with microvascular complications were not independent of other risk factors.

Keywords CHD - Complications - Coronary heart disease . Fibrinogen · Macrovascular · Microvascular · Sialic acid .

Type 1 diabetes
Abbreviations
CRP C-reactive protein
HR hazard ratio
PCS Prospective Complications Study 
sHR standardised hazard ratio

sOR standardised odds ratio

\section{Introduction}

Type 1 diabetes is associated with a four- (in men) to eightfold (in women) increased risk of CHD, compared with the general population $[1,2]$. This excess CHD risk in type 1 diabetes patients is not fully explained by established risk factors. It is also not clear what explains the abolition of a sex-related difference in CHD risk for patients with type 1 diabetes, with women being at equally high risk of developing CHD as men [3]. Concentrations of the inflammatory markers C-reactive protein (CRP) and fibrinogen are raised in patients with type 2 diabetes and found to be independently related to cardiovascular morbidity and mortality in these patients [4-7]. There is evidence that inflammation precedes atherosclerosis and inflammation may be the common antecedent of atherosclerosis and type 2 diabetes [8]. Sialic acid and fibrinogen are inflammatory markers that are highly correlated with CRP and could be as important as CRP in the prediction of CHD.

Sialic acid is the terminal sugar of the oligosaccharide chain of acute-phase proteins. It is a molecule attached to acute-phase proteins, such as fibrinogen, which is why both inflammatory markers are closely related.

In the general population, elevated levels of sialic acid have been shown to be a strong predictor of CHD [9]. In type 2 diabetic patients, associations between sialic acid and CHD have been shown predominantly in men [10]. Since patients with type 1 diabetes have a higher risk of developing CHD, sialic acid and/or fibrinogen could potentially explain this increased risk. For fibrinogen, initial associations with incident CHD in people with type 2 diabetes have been shown, but multivariable analyses indicated these findings were non-significant $[4,11,12]$. For microvascular complications, cross-sectional studies have shown elevated plasma sialic acid concentrations in patients with type 1 and type 2 diabetes and retinopathy $[13,14]$ or albuminuria $[13,15-17]$ compared with people without these complications. The cross-sectional analyses of the EURODIAB study showed elevated sialic acid concentrations in type 1 diabetic patients with retinopathy (men), neuropathy (men) and albuminuria (men and women), indicating the need to explore these findings prospectively [18].

To the best of our knowledge, these inflammatory markers have not been prospectively examined in relation to a wide range of vascular complications in patients with type 1 diabetes. Therefore, we examined whether fibrinogen and sialic acid are related to incident micro- and macrovascular complications in patients with type 1 diabetes.

\section{Methods}

Study design and participants A subset of the EURODIAB Prospective Complications Study (PCS) was used. Full details of the methods in the EURODIAB PCS have been published elsewhere [19-21]. The original study contained 3,250 patients with type 1 diabetes, aged between 15 and 60 years and recruited from 31 centres in 16 European countries. Type 1 diabetes was defined as diabetes diagnosed before the age of 36 years with a continuous need for insulin within 1 year of diagnosis. Of those invited, $85 \%$ participated. Those with a duration of diabetes of less than 1 year and pregnant women were excluded. Ethics committee approval was obtained at each centre and all patients provided written informed consent.

Measurements of risk factors All risk factors and microvascular complications were measured at baseline (between 1989 and 1991) according to a standardised protocol [22]. Blood pressure was recorded twice with a random zero sphygmomanometer (Hawskley, Lancing, UK). Hypertension was defined as a systolic blood pressure $\geq 140 \mathrm{mmHg}$ or diastolic blood pressure $\geq 90 \mathrm{mmHg}$ and/or the use of blood pressure-lowering drugs. WHR and BMI $\left(\mathrm{kg} / \mathrm{m}^{2}\right)$ were calculated.

Laboratory measurements A single $24 \mathrm{~h}$ urine collection was performed to calculate AER at baseline after excluding proteinuria due to urinary tract infection. Urinary albumin was measured in a single laboratory by an immunoturbidimetric method (Sanofi Diagnostics Pasteur, Minneapolis, MN, USA) [23]. A blood sample was taken to measure plasma lipids (fasting triacylglycerol, cholesterol, HDLcholesterol) and $\mathrm{HbA}_{1 \mathrm{c}}$. Plasma levels of triacylglycerol [24] and cholesterol [25] and HDL-cholesterol [26] were assayed by standard enzymatic methods (Boehringer Mannheim, Lewes, UK) using a centrifugal analyser (CobasBio; Roche, Welwyn Garden City, UK). For HDL, samples with triacylglycerol concentrations $>3 \mathrm{mmol} / \mathrm{l}$ were diluted with $0.15 \mathrm{~mol} / \mathrm{l}$ sodium chloride solution before chemical precipitation. All analyses were performed centrally. LDLcholesterol was calculated from Friedewald's formula if triacylglycerol values were below $4 \mathrm{mmol} / \mathrm{l}$ [27]. Non-HDLcholesterol was calculated as HDL-cholesterol subtracted from total cholesterol. $\mathrm{HbA}_{1 \mathrm{c}}$ was measured centrally by an enzyme immunoassay (Dako, Ely, UK) using a monoclonal antibody [28].

Fibrinogen $(n=1515)$ was assayed by a clotting assay based on the prothrombin time, using an autoanalyser (Series 300; Instrumentation Laboratories, Lexington, MA, USA) [29]. A pooled plasma standard, calibrated against the UK standard (UK National Institute for Biological Standards and 
Control, South Mimms, UK), was employed. Hidden duplicate samples were included to check on reproducibility, with satisfactory performance recorded according to the UK National Quality Assurance guidelines. The interassay CV was $5-8 \%$ and the intra-assay $\mathrm{CV} 4-7 \%$. The reference range of fibrinogen in the general population was between 5.6 and $11.2 \mu \mathrm{mol} / \mathrm{l}$. Fibrinogen levels in $\mathrm{g} / \mathrm{l}$ were converted into SI units $(\mu \mathrm{mol} / \mathrm{l})$ by multiplying by 2.94 .

Total plasma sialic acid $(n=1113)$ was assayed using an enzymatic method (Boehringer Mannheim), adapted for use on a centrifugal analyser (Cobas Bio or Fara; Roche). The between-batch coefficient of variation of the assay was 3.8\% [30]. The range for sialic acid in the general population was between $1.6-2.2 \mathrm{mmol} / \mathrm{l}$.

The difference in numbers at baseline $(n=1515$ for fibrinogen, $n=1113$ for sialic acid) was due to the fact that two separate laboratories were involved at different times (fibrinogen first in Sheffield [31] and sialic acid [18] later at Guy's Hospital, London, UK).

Outcome All complications from albuminuria, retinopathy, neuropathy and CHD were assessed during follow-up. Albuminuria was defined as an AER above $20 \mu \mathrm{g} / \mathrm{min}$. At baseline, AER was measured once, whereas at follow-up it was measured twice, with the mean taken as data.

Retinopathy was assessed by retinal photographs taken according to the EURODIAB protocol [32]. Grading was performed by the Retinopathy Grading Centre at the Hammersmith Hospital, Imperial College London. Any retinopathy contained both non-proliferative (levels 1-3) and proliferative retinopathy (levels 4 and 5).

Total neuropathy was assessed combining peripheral neuropathy and autonomic neuropathy. Peripheral neuropathy was assessed with neuropathic symptoms and signs, including measurement of vibration perception threshold. Autonomic neuropathy was defined as an RR ratio (the ratio of the longest electrocardiographic $R R$ interval between the 28th and 32nd beats after standing to the shortest interval between the 13th and 17th beats) of $<1.04$ and/or a fall in blood pressure from resting to standing of $>20 \mathrm{mmHg}[33,34]$.

CHD was defined as a previous physician diagnosis of CHD or presence of ECG abnormalities. The former included: myocardial infarction, angina pectoris or coronary artery bypass graft surgery. A conventional 12-lead resting ECG was recorded on each participant. ECG abnormalities were classified by two observers according to the Minnesota Code [35]. Any discrepancies between the two observers were adjudicated by a third. ECG abnormalities suggestive of probable ischaemia consist of codes 1.1 and 1.2 (major Q/QS waves) and code 7.1 (complete left bundle branch block). Possible ischaemia consists of code 1.3 (minor Q waves); codes 4.1, 4.2 and 4.3 (ST segment abnormalities); and codes 5.1, 5.2 and
5.3 (T wave abnormalities). Death due to CHD causes was also included, coded according to the International Classification of Diseases (9th revision) classification (410-414; available from: ftp://ftp.cdc.gov/pub/Health_Statistics/NCHS/Publications/ ICD-9/, last accessed in December 2007).

All CHD events were captured by questionnaire with additional supporting information from hospital records, death certificates and other healthcare documents.

Statistical analysis The statistical packages SAS (SAS 8.0; SAS, Cary, NC, USA) and STATA 7 (Stata Statistical Software, Release 7.0; Stata Corporation, College Station, TX, USA) were used to perform all analyses. Spearman rank correlations were used to test cross-sectional relationships between risk factors. Non-normally distributed variables were log-transformed. For each of the outcomes, those with baseline micro- or macrovascular complications were excluded according to which outcome was analysed.

For the CHD analyses, 681 of the 3,250 patients at baseline could not be assessed or were excluded from follow-up because they did not meet the inclusion criteria at baseline or had CHD at that time point. Of the remaining 2,569 patients, 2,329 were assessed for CHD, of whom 151 had CHD at follow-up and 2,178 did not.

For albuminuria, 1,134 patients were available without albuminuria at baseline, 972 of whom remained normoalbuminuric throughout the follow-up, while 162 developed incident albuminuria. For retinopathy, 767 patients without baseline retinopathy were available. Of these, 428 developed incident retinopathy. For neuropathy, 1,172 patients without baseline neuropathy were available. Of these, 276 developed neuropathy and 896 did not.

To test whether the relationship between inflammatory markers and CHD was linear, a quadratic term was fitted for sialic acid and for fibrinogen. Likelihood ratio tests were performed to test differences between a model including a linear term and quadratic term, and a model with the linear term. Evidence of non-linearity was accepted at $p<0.05$.

For CHD, time-to-event data were available and therefore univariable and multivariable Cox proportional hazards modelling was used to estimate standardised hazard ratios (sHR) for CHD associations with the inflammatory markers. sHR were estimated from these models as follows: Exp $(\beta \times \mathrm{SD})$. Interactions were tested by fitting sex $\times$ sialic acid and sex $\times$ fibrinogen interaction terms in the Cox proportional hazards models and comparing a model with sialic acid, sex and one interaction term with the crude model (sialic acid alone).

For microvascular outcomes, no time-to-event data were available, but presence or absence of each complication was assessed during the 1997-1999 follow-up visit examinations. Therefore, univariable and multivariable logistic regression analyses were used to analyse relationships between inflam- 
matory markers and microvascular complications. Again, standardised odds ratios (sORs) were presented.

\section{Results}

Sialic acid, fibrinogen and CHD The total study population consisted of 2,329 patients, of whom 151 developed CHD. Sialic acid and fibrinogen were measured at baseline (1989-1991) in subsets of the total population. The mean follow-up period was 7 years. The baseline risk factors of those included and those lost to follow-up are given in Table 1 . In the same table, baseline characteristics between those with and without sialic acid (or fibrinogen) measurements are compared. The mean age was 32 years (SD 10, range 15-61) and duration of diabetes 14 years (SD 9, range 1-56). Those lost to follow-up had a markedly greater atherogenic risk factor profile than those included in the study. The sialic acid subset $(n=1113)$ differed significantly from those without sialic acid measurements $(n=$ 1216), with an increased duration of diabetes, systolic blood pressure, BMI in women, albumin excretion rate and insulin dose, as well as a higher number of complications from hypertension, retinopathy and albuminuria; there were also more men. The patients with fibrinogen measurements $(n=1515)$ did not differ significantly in terms of any of the risk factors compared with participants without these measurements.

Sialic acid concentrations were significantly raised in those with CHD compared with those without. Stratified by

Table 1 Baseline risk factors in the EURODIAB PCS type 1 diabetic population, including those lost to follow-up and patients with sialic acid and fibrinogen measurements

\begin{tabular}{|c|c|c|c|c|}
\hline & $\begin{array}{l}\text { Total population followed } \\
n=2,329\end{array}$ & $\begin{array}{l}\text { Lost-to-follow-up } \\
n=921\end{array}$ & $\begin{array}{l}\text { Sialic acid subset } \\
n=1,113\end{array}$ & $\begin{array}{l}\text { Fibrinogen subset } \\
n=1,515\end{array}$ \\
\hline Age (years) & $32.1 \pm 9.7$ & $34.2 \pm 11.3^{\dagger \dagger \dagger}$ & $32.2 \pm 9.7$ & $32.0 \pm 9.5$ \\
\hline Diabetes duration (years) & $14.3 \pm 9.0$ & $15.7 \pm 10.0^{\dagger \dagger \dagger}$ & $14.9 \pm 9.0 * *$ & $14.1 \pm 9.0$ \\
\hline $\mathrm{HbA}_{1 \mathrm{c}}(\%)^{\mathrm{a}}$ & $8.36 \pm 1.92$ & $8.65 \pm 1.93^{\dagger \dagger \dagger}$ & $8.43 \pm 1.93$ & $8.36 \pm 1.93$ \\
\hline Systolic BP (mmHg) & $120.2 \pm 16.8$ & $124.2 \pm 19.9^{\dagger \dagger \dagger}$ & $121.2 \pm 17.0 * *$ & $120.7 \pm 16.7$ \\
\hline Diastolic BP (mmHg) & $75.0 \pm 11.2$ & $76.7 \pm 11.9^{\dagger \dagger \dagger}$ & $75.1 \pm 11.3$ & $75.0 \pm 11.4$ \\
\hline Cholesterol (mmol/l) & $5.31 \pm 1.13$ & $5.37 \pm 1.19$ & $5.32 \pm 1.14$ & $5.29 \pm 1.11$ \\
\hline LDL-cholesterol (mmol/l) & $3.33 \pm 0.98$ & $3.44 \pm 1.03^{\dagger}$ & $3.34 \pm 1.00$ & $3.34 \pm 0.97$ \\
\hline HDL-cholesterol (mmol/l) & $1.50 \pm 0.43$ & $1.38 \pm 0.44^{\dagger \dagger \dagger}$ & $1.50 \pm 0.44$ & $1.51 \pm 0.42$ \\
\hline Non HDL-cholesterol (mmol/1) & $3.81 \pm 1.14$ & $3.99 \pm 1.20^{\dagger \dagger \dagger}$ & $3.81 \pm 1.15$ & $3.78 \pm 1.12$ \\
\hline Fasting triacylglycerol $(\mathrm{mmol} / \mathrm{l})$ & $0.89(0.69-1.26)$ & $1.06(0.78-1.58)^{\dagger \dagger \dagger}$ & $0.91(0.70-1.30)$ & $0.88(0.68-1.20)$ \\
\hline \multicolumn{5}{|l|}{ Waist circumference $(\mathrm{cm})(\mathrm{M} / \mathrm{F})$} \\
\hline Men & $83.4 \pm 8.7$ & $83.9 \pm 9.9$ & $83.5 \pm 8.5$ & $83.3 \pm 8.6$ \\
\hline Women & $75.5 \pm 8.6$ & $78.9 \pm 12.1^{\dagger \dagger}$ & $75.9 \pm 8.9$ & $75.4 \pm 8.6$ \\
\hline \multicolumn{5}{|l|}{ WHR (M/F) } \\
\hline Men & $0.88 \pm 0.08$ & $0.89 \pm 0.09^{\dagger}$ & $0.88 \pm 0.08$ & $0.88 \pm 0.08$ \\
\hline Women & $0.80 \pm 0.11$ & $0.82 \pm 0.13^{\dagger \dagger \dagger}$ & $0.80 \pm 0.12$ & $0.80 \pm 0.11$ \\
\hline \multicolumn{5}{|l|}{ BMI $\left(\mathrm{kg} / \mathrm{m}^{2}\right)(\mathrm{M} / \mathrm{F})$} \\
\hline Men & $23.6 \pm 2.6$ & $23.4 \pm 2.9$ & $23.5 \pm 2.7$ & $23.6 \pm 2.6$ \\
\hline Women & $23.3 \pm 2.9$ & $23.8 \pm 3.5^{\dagger \dagger}$ & $23.5 \pm 3.0^{*}$ & $23.3 \pm 2.9$ \\
\hline $\operatorname{AER}(\mu \mathrm{g} / \mathrm{min})$ & $10.9(6.6-23.6)$ & $11.8(6.2-34.4)^{\dagger \dagger}$ & $13.1(7.5-34.6)^{* * *}$ & $11.0(6.7-24.2)$ \\
\hline Insulin dose $\left(\mathrm{U}^{\text {day }}{ }^{-1} \mathrm{~kg}^{-1}\right)$ & $0.67(0.54-0.81)$ & $0.63(0.51-0.78)^{\dagger \dagger \dagger}$ & $0.68(0.55-0.83)^{* *}$ & $0.67(0.55-0.82)$ \\
\hline Insulin injections per day & $2.46 \pm 0.57$ & $2.47 \pm 0.57$ & $2.48 \pm 0.58$ & $2.45 \pm 0.57$ \\
\hline Men & $52(1,202)$ & $51(466)$ & $54(603)^{*}$ & $51(771)$ \\
\hline Hypertension (yes) & $21(493)$ & $32(288)^{\dagger \dagger \dagger}$ & $23(258)^{*}$ & $22(328)$ \\
\hline BP-lowering drugs (yes) & $8(189)$ & $15(138)^{\dagger \dagger \dagger}$ & $10(113)^{* *}$ & $8(122)$ \\
\hline Lipid-lowering drugs (yes) & $1.3(31)$ & $1.7(15)$ & $1.4(16)$ & $1.3(20)$ \\
\hline Current smoking & $31(719)$ & $35(316)^{\dagger}$ & $32(352)$ & $30(460)$ \\
\hline Ex-smoking & $18(414)$ & $18(162)$ & $18(202)$ & $18(275)$ \\
\hline Retinopathy & $45(864)$ & $50(280)^{\dagger \dagger}$ & $52(468)^{* * *}$ & $45(560)$ \\
\hline Albuminuria & $29(643)$ & $35(292)^{\dagger \dagger \dagger}$ & $38(418)^{* * *}$ & $29(433)$ \\
\hline Neuropathy & $32(722)$ & $48(427)^{\dagger+\dagger}$ & $33(363)$ & $31(460)$ \\
\hline
\end{tabular}

Values are means $\pm \mathrm{SD}$, median (interquartile range) or $\%(n)$

${ }^{\mathrm{a}} \mathrm{HbA}_{1 \mathrm{c}}$ values corrected according to the DCCT method

$* p<0.05, * * p \leq 0.01, * * * p \leq 0.001$ for differences between participants with sialic acid measurements and those without (1,113 vs 1,216 patients) ${ }^{\dagger} p<0.05,{ }^{\dagger \dagger} p \leq 0.01,{ }^{\dagger \dagger} p \leq 0.001$ for differences between participants lost to follow-up and those included (921 vs 2,329 patients) 
sex, this difference was only statistically significant in men (Table 2). Moreover, the baseline sialic acid concentration differed by $\operatorname{sex}(p=0.04$ for sialic acid $\times$ sex interaction), with a significantly higher concentration in women than in men (mean concentration 2.01 and $1.89 \mathrm{mmol} / 1$ respectively, women vs men, $p<0.0001$; Table 2 ). The results were similar for the relationship between fibrinogen and CHD, which was statistically significant only in men. Again, there was a statistically significant fibrinogen $\times$ sex interaction $(p=0.005)$, with a higher concentration in type 1 diabetic women than in men (mean $9.90 \mu \mathrm{mol} / 1$ and $8.99 \mu \mathrm{mol} / 1$ respectively, women vs men, $p<0.0001$ ).

The Spearman rank correlation coefficients between risk factors and sialic acid or fibrinogen stratified by sex are given in the Electronic supplementary material (ESM) Table 1, calculated to establish potential confounders. Statistically significant positive correlations were found between sialic acid and duration of diabetes, $\mathrm{HbA}_{1 \mathrm{c}}$, lipid levels (apart from HDL-cholesterol which was negative), WHR and albumine/creatinine ratio. For fibrinogen, similar correlations were found for most risk factors, but statistically significant positive correlations were also found with age, BMI and both systolic and diastolic blood pressure. The correlation between fibrinogen and sialic acid was $0.32(p \leq 0.0001 ; 0.33$ in men, 0.27 in women). Stronger correlations were found in men than in women with inflammatory markers, especially for lipids and obesity measures. The relationship between current smoking and sialic acid was stronger in men than in women (mean sialic acid concentration 1.95 vs $1.85 \mathrm{mmol} / \mathrm{l}, p=$ 0.0005 , in men; 2.0 vs $2.0 \mathrm{mmol} / 1, p=0.8$, in women smokers vs non-smokers). For fibrinogen, by contrast, the relationship was stronger in women than in men for smokers vs non-smokers (mean fibrinogen levels 9.22 vs $8.88 \mu \mathrm{mol} / \mathrm{l}$, $p=0.1$, in men; 10.27 vs $9.76 \mu \mathrm{mol} / 1, p=0.03$, in women).

Linearity was checked for the relationships between inflammatory markers and CHD. There was no evidence of a non-linear relationship ( $p=0.09$ for sialic acid and $p=0.4$ for fibrinogen in likehood ratio test). There was also no evidence of non-linearity when findings were stratified by sex. The linearity of the relationship was shown by the increasing hazard ratios (HRs) with quartiles of sialic acid concentrations. Thus HRs changed from 1.0 to 4.7 ( $p=$ $0.047)$ and from $5.9(p=0.02)$ to $8.3(p=0.006)$ respectively for sialic acid levels $<1.7,1.7-1.9,1.9-2.2$ and $\geq 2.2 \mathrm{mmol} / \mathrm{l}$.

The main analyses for CHD are shown in Table 3. In univariable models, an increase in one SD unit of baseline sialic acid concentration was related to a 1.6-fold increased risk of CHD, but only for men. Similarly, for fibrinogen, a sHR of 1.7 was found, but only for men. In women, no statistically significant association between sialic acid or fibrinogen and CHD was observed. In multivariable models, there was still a clear 1.5-fold increased risk of CHD with increasing concentrations of sialic acid when adjusted for age, diabetes duration, $\mathrm{HbA}_{1 \mathrm{c}}$, triacylglycerol, WHR, current smoking, systolic blood pressure and AER, but only in men. Similarly, for fibrinogen, there was a 1.4-fold increased risk of CHD in multivariable models, also only in men. Since albuminuria is an important risk marker for CHD, adjustment for this marker attenuated the relationship between fibrinogen and CHD. Smoking did not affect the relationship between fibrinogen and CHD. Adjustment for centre and the use of blood pressure-lowering or lipidlowering drugs did not alter the results.

Sialic acid, fibrinogen and microvascular complications Statistically significant univariable associations were shown between: (1) sialic acid and incident retinopathy in men (sOR 1.68, 95\% CI 1.10-2.57); (2) fibrinogen and retinopathy in women (sOR 1.37, 95\% CI 1.06-1.78); and (3) sialic acid and neuropathy in men (sOR $1.37,95 \%$ CI 1.06-1.77; Table 4). These associations, however, became non-significant in multivariable models (adjusting for age, diabetes duration and $\mathrm{HbA}_{1 \mathrm{c}}$ ). There was no relationship between sialic acid or fibrinogen with albuminuria in univariable analyses.

\section{Discussion}

Sialic acid and fibrinogen are significant predictors of CHD in men with type 1 diabetes. These relationships are independent of established risk factors, supporting the hypothesis

Table 2 Unadjusted baseline sialic acid and fibrinogen concentrations by CHD status per group at follow-up

\begin{tabular}{|c|c|c|c|c|c|c|}
\hline & \multicolumn{2}{|l|}{ Total group } & \multicolumn{2}{|l|}{ Men } & \multicolumn{2}{|l|}{ Women } \\
\hline & CHD & No CHD & CHD & No CHD & CHD & No CHD \\
\hline Sialic acid concentrations available $(n)$ & 68 & 1,045 & 31 & 572 & 34 & 473 \\
\hline Sialic acid $(\mathrm{mmol} / \mathrm{l})$ & $2.06 \pm 0.36^{* *}$ & $1.94 \pm 0.38$ & $2.09 \pm 0.35^{* * *}$ & $1.88 \pm 0.35$ & $2.04 \pm 0.38$ & $2.01 \pm 0.40$ \\
\hline Fibrinogen concentrations available $(n)$ & 92 & 1,423 & 46 & 725 & 46 & 698 \\
\hline Fibrinogen $(\mu \mathrm{mol} / 1)$ & $10.06 \pm 3.26^{*}$ & $9.40 \pm 2.75$ & $10.37 \pm 3.64 * * *$ & $8.90 \pm 2.63$ & $9.74 \pm 2.84$ & $9.91 \pm 2.79$ \\
\hline
\end{tabular}

Values are means $\pm \mathrm{SD}$

${ }^{*} p<0.05, * * p \leq 0.01, * * * p \leq 0.001$ vs corresponding control 
Table 3 Risk of CHD by 1 SD increase in inflammatory markers

\begin{tabular}{|c|c|c|c|c|}
\hline & \multicolumn{2}{|l|}{ Sialic acid } & \multicolumn{2}{|l|}{ Fibrinogen } \\
\hline & CHD in men & CHD in women & CHD in men & CHD in women \\
\hline$n$ & 603 & 510 & 771 & 744 \\
\hline Events $(n)$ & 31 & 37 & 46 & 46 \\
\hline Crude model & $1.59(1.21-2.09)^{* * *}$ & $1.04(0.77-1.41)$ & $1.69(1.32-2.16)^{* * *}$ & $0.99(0.74-1.31)$ \\
\hline Adjustment $1^{\mathrm{a}}$ & $1.64(1.22-2.21)^{* * *}$ & $1.07(0.78-1.49)$ & $1.50(1.16-1.95)^{* *}$ & $1.00(0.75-1.33)$ \\
\hline Adjustment $2^{\mathrm{b}}$ & $1.50(1.05-2.15)^{*}$ & $0.95(0.67-1.35)$ & $1.23(0.88-1.70)$ & $0.81(0.61-1.09)$ \\
\hline Adjustment $3^{\mathrm{c}}$ & & & $1.40(1.06-1.86)^{* *}$ & $0.90(0.66-1.21)$ \\
\hline
\end{tabular}

Unless otherwise indicated, values are sHR $(95 \% \mathrm{CI})$

${ }^{\mathrm{a}}$ Adjusted for age and diabetes duration

${ }^{\mathrm{b}}$ Adjusted for $\mathrm{HbA}_{1 \mathrm{c}}$, triacylglycerol, WHR, smoking, systolic blood pressure and AER

${ }^{\mathrm{c}}$ Adjusted for $\mathrm{HbA}_{1 \mathrm{c}}$, triacylglycerol, WHR, smoking, systolic blood pressure, but not AER

${ }^{*} p<0.05,{ }^{*} p \leq 0.01, * * * p \leq 0.001$

that inflammation plays a role in the development of CHD. The associations found with microvascular complications were explained by other risk factors.

The reasons for a difference in the relationship between sialic acid or fibrinogen and CHD in men and women are unknown, but this difference could explain the abolition of a sex-related difference in CHD risk in people with type 1 diabetes. The levels of both inflammatory markers were higher in women than in men with type 1 diabetes, but not outside the reference range in the general population. The levels of sialic acid and fibrinogen were significantly higher in patients with CHD than in those without and were at the upper end of the normal range. It is not entirely clear why sialic acid and fibrinogen are related to CHD only in men. This relation has been found previously in a cross-sectional analysis of the Guy's and Lewisham Hospitals Surveys, where the main conclusion was that there were univariate associations between elevated sialic acid concentrations and CHD in men (but not women) with type 2 diabetes [10]. The finding that the concentrations of sialic acid and fibrin- ogen were statistically significantly higher in women than in men with type 1 diabetes indicates different mechanisms in the two sexes.

It could be said that sialic acid and fibrinogen do not constitute a component cause of CHD development in women, because there may be other essential causes, which are more important. The correlations between the inflammatory markers and lipids, obesity measures and smoking were stronger in men than in women, but adjusting for these would diminish the relationship between these inflammatory markers and CHD in men. This strong association persisted, however, after adjustment for all these factors, so this could not explain the sex-related difference. Another possible explanation for the predictive value of inflammatory markers in men, but not in women, could be the possibility that the relationship between sialic acid or fibrinogen and CHD was not linear in women, with a flattening of the relationship towards the end. In this case, the CHD risk would not increase any longer. However, this was not true in our data, since for men and women there was no evidence of non-linearity. Sialic

Table 4 Risk of developing albuminuria, retinopathy and neuropathy during the follow-up period by 1 SD unit increase in inflammatory markers

\begin{tabular}{|c|c|c|c|c|}
\hline & \multicolumn{2}{|l|}{ Sialic acid } & \multicolumn{2}{|l|}{ Fibrinogen } \\
\hline & Men & Women & Men & Women \\
\hline Albuminuria, crude model & $0.94(0.67-1.31)$ & $1.28(0.83-1.96)$ & $0.92(0.67-1.26)$ & $1.30(0.98-1.73)$ \\
\hline \multicolumn{5}{|l|}{ Retinopathy } \\
\hline Crude model & $1.68(1.10-2.57)^{* *}$ & $1.17(0.87-1.56)$ & $1.05(0.81-1.37)$ & $1.37(1.06-1.78)^{*}$ \\
\hline Adjusted for age and diabetes duration & $1.61(1.04-2.48)^{*}$ & $1.11(0.82-1.50)$ & $1.06(0.80-1.41)$ & $1.39(1.06-1.81)^{*}$ \\
\hline Adjusted as above plus for $\mathrm{HbA}_{1 \mathrm{c}}$ & $1.43(0.91-2.23)$ & & & $1.28(0.96-1.71)$ \\
\hline \multicolumn{5}{|l|}{ Neuropathy } \\
\hline Crude model & $1.37(1.06-1.77)^{*}$ & $0.93(0.70-1.23)$ & $1.25(0.97-1.62)$ & $0.88(0.69-1.11)$ \\
\hline Adjusted for age and diabetes duration & $1.36(1.04-1.77)^{*}$ & $0.96(0.72-1.28)$ & $1.15(0.88-1.51)$ & $0.87(0.68-1.10)$ \\
\hline Adjusted as above plus for $\mathrm{HbA}_{1 \mathrm{c}}$ & $1.26(0.96-1.66)$ & & & \\
\hline
\end{tabular}

Values are sOR $(95 \% \mathrm{CI})$

${ }^{*} p<0.05,{ }^{*} p \leq 0.01$ 
acid is the terminal sugar of the oligosaccharide chain of some glycoproteins, particularly some of the acute-phase proteins and is (biologically) bound to fibrinogen. We found that the results for fibrinogen and sialic acid were similar, which adds weight to our conclusion that acute-phase or inflammatory markers are strong predictors of CHD risk in type 1 diabetes.

In the general population, a large Swedish study with a 20 year follow-up reported a 1.9-fold increased CHD mortality risk in 50,000 non-diabetic men and women with increased concentrations of sialic acid [9]. More recently, the 17 year prospective Busselton (WA, Australia) Health Survey of 1,612 non-diabetic men and women reported that a $0.8 \mathrm{mmol} / 1$ increase in sialic acid was significantly associated with a HR of 1.21 (95\% CI 1.02-1.45) for CHD events, with men having an HR of 1.06 (95\% CI $0.82-1.37)$ and women an HR of 1.40 (95\% CI 1.11-1.76) [36]. The evidence of a relationship between sialic acid and CHD in patients with type 1 or type 2 diabetes is scarce. A 13 year follow-up study in type 2 diabetes found that sialic acid or 'activator of the innate immune system' was a strong independent predictor of CVD mortality in patients with type 2 diabetes [8]. We have now found that this 'activation of the innate immune system' is also present in patients with type 1 diabetes and highly predictive of CHD.

The relationship between sialic acid and incident microvascular complications did not seem to be very strong in our data, especially when analysed in multivariable models. Previously it has been shown that elevated sialic acid concentrations were cross-sectionally associated with diabetic retinopathy in patients with type 1 [13] and type 2 diabetes [14]. These studies used small study populations and no adjustment for confounders was carried out. For albuminuria, several studies have found a positive relationship with sialic acid in patients with type 1 diabetes [13, 16, 17], including one prospective study assessing sialic acid levels 3 years before diagnosis of microalbuminuria [37]. It is conceivable that sialic acid concentration plays a role only in early diabetic nephropathy $[17,37]$, possibly explaining why we failed to find any relationship with albuminuria after 7 years. For neuropathy, one study in type 1 diabetes showed no relationship with sialic acid [13].

There is extensive evidence from prospective studies and meta-analyses in the general population that fibrinogen predicts CHD in individuals with and without prior vascular disease, with relative risks of around 2 being reported [38-41]. In patients with type 1 or type 2 diabetes, the evidence is less clear. Elevated concentrations of fibrinogen have been demonstrated in patients with type 2 diabetes $[11,12,42]$, but the relationship between fibrinogen and CVD seemed to be diminished after further risk factor adjustments [4, 11]. Both of these studies adjusted for a wide range of risk factors, but not for albuminuria. We have demonstrated that fibrinogen also predicts CHD in patients with type 1 diabetes, although this association was no longer statistically significant after further adjustment for albuminuria. Because fibrinogen levels can be reduced considerably by lifestyle interventions (such as physical exercise, smoking cessation and moderate alcohol consumption) that also affect levels of established risk factors, the possibility of measuring fibrinogen in order to prevent or predict future CHD is clearly of interest. In our data, although participants who smoked had higher levels of fibrinogen than none smokers, adjustment for smoking did not seem to affect the relationship between fibrinogen and CHD in any way.

The EURODIAB PCS offers the possibility to study complications of type 1 diabetes, as it provides a large, multicentre, European cohort of patients attending a clinic at least once a year. In addition, all centres used similar standardised methods. In the current study enough coronary events have accrued over a long follow-up period to carry out powerful analyses. However, because some centres did not participate in these measurements and samples were therefore not available, a large number of measurements of sialic acid were not available. Participants lost-to-follow-up had a slightly more atherogenic profile, which could have led to overestimation of the true association between sialic acid or fibrinogen and CHD incidence.

The study question was decided upon a priori and the relevant statistical tests were applied accordingly, not testing for every conceivable association. Adjustment for centre did not affect results. Residual confounding could have played a role, since all baseline variables were measured only once, thereby possibly underestimating the role of confounding. Nevertheless, strong associations were found for sialic acid and fibrinogen, indicating that residual confounding could not wholly account for our findings.

In conclusion, in this large prospective Europe-wide cohort study of patients with type 1 diabetes, sialic acid and fibrinogen were found to be significant and independent predictors of CHD in men, supporting the hypothesis that inflammation plays a role in the development of CHD. No independent associations were found with microvascular complications. The finding that both of these inflammatory markers are related to CHD only in men needs to be explored further.

Acknowledgements The EURODIAB PCS was supported by grants from the Wellcome Trust, the European Community and Diabetes UK.

Duality of interest The authors declare that there is no duality of interest associated with this manuscript.

Open Access This article is distributed under the terms of the Creative Commons Attribution Noncommercial License which permits any noncommercial use, distribution, and reproduction in any medium, provided the original author(s) and source are credited. 


\section{References}

1. Laing SP, Swerdlow AJ, Slater SD et al (2003) Mortality from heart disease in a cohort of 23,000 patients with insulin-treated diabetes. Diabetologia 46:760-765

2. Soedamah-Muthu SS, Fuller JH, Mulnier HE et al (2006) High risk of cardiovascular disease in patients with type 1 diabetes in the U.K.: a cohort study using the general practice research database. Diabetes Care 29:798-804

3. Barrett-Connor EL, Cohn BA, Wingard DL, Edelstein SL (1991) Why is diabetes mellitus a stronger risk factor for fatal ischemic heart disease in women than in men? The Rancho Bernardo Study. JAMA 265:627-631

4. Saito I, Folsom AR, Brancati FL et al (2000) Nontraditional risk factors for coronary heart disease incidence among persons with diabetes: the Atherosclerosis Risk in Communities (ARIC) Study. Ann Intern Med 133:81-91

5. Jager A, van Hinsbergh VW, Kostense PJ et al (2000) Increased levels of soluble vascular cell adhesion molecule 1 are associated with risk of cardiovascular mortality in type 2 diabetes: the Hoorn study. Diabetes 49:485-491

6. Jager A, van Hinsbergh VW, Kostense PJ et al (1999) von Willebrand factor, C-reactive protein, and 5-year mortality in diabetic and nondiabetic subjects: the Hoorn Study. Arterioscler Thromb Vasc Biol 19:3071-3078

7. Hoogeveen EK, Kostense PJ, Jakobs C et al (2000) Hyperhomocysteinemia increases risk of death, especially in type 2 diabetes: 5-year follow-up of the Hoorn Study. Circulation 101:1506-1511

8. Pickup JC, Mattock MB (2003) Activation of the innate immune system as a predictor of cardiovascular mortality in type 2 diabetes mellitus. Diabet Med 20:723-726

9. Lindberg G, Rastam L, Gullberg B, Eklund GA (1992) Serum sialic acid concentration predicts both coronary heart disease and stroke mortality: multivariate analysis including 54,385 men and women during 20.5 years follow-up. Int J Epidemiol 21:253-257

10. Pickup JC, Mattock MB, Crook MA et al (1995) Serum sialic acid concentration and coronary heart disease in NIDDM. Diabetes Care 18:1100-1103

11. Kannel WB, D'Agostino RB, Wilson PW, Belanger AJ, Gagnon DR (1990) Diabetes, fibrinogen, and risk of cardiovascular disease: the Framingham experience. Am Heart J 120:672-676

12. Ganda OP, Arkin CF (1992) Hyperfibrinogenemia. An important risk factor for vascular complications in diabetes. Diabetes Care $15: 1245-1250$

13. Powrie JK, Watts GF, Crook MA et al (1996) Serum sialic acid and the long-term complications of insulin-dependent diabetes mellitus. Diabet Med 13:238-242

14. Crook MA, Tutt P, Pickup JC (1993) Elevated serum sialic acid concentration in NIDDM and its relationship to blood pressure and retinopathy. Diabetes Care 16:57-60

15. Chen J, Gall MA, Yokoyama H et al (1996) Raised serum sialic acid concentration in NIDDM patients with and without diabetic nephropathy. Diabetes Care 19:130-134

16. Crook MA, Earle K, Morocutti A et al (1994) Serum sialic acid, a risk factor for cardiovascular disease, is increased in IDDM patients with microalbuminuria and clinical proteinuria. Diabetes Care 17:305-310

17. Yokoyama H, Jensen JS, Jensen T, Deckert T (1995) Serum sialic acid concentration is elevated in IDDM especially in early diabetic nephropathy. J Intern Med 237:519-523

18. Crook MA, Pickup JC, Lumb PJ et al (2001) Relationship between plasma sialic acid concentration and microvascular and macrovascular complications in type 1 diabetes: the EURODIAB Complications Study. Diabetes Care 24:316-322
19. EURODIAB IDDM Complications Study group (1994) Microvascular and acute complications in IDDM patients: the EURODIAB IDDM Complications Study. Diabetologia 37:278-285

20. Chaturvedi N, Sjoelie AK, Porta M et al (2001) Markers of insulin resistance are strong risk factors for retinopathy incidence in type 1 diabetes: The EURODIAB PCS. Diabetes Care 24:284-289

21. Chaturvedi N, Bandinelli S, Mangili R et al (2001) Microalbuminuria in type 1 diabetes: rates, risk factors and glycemic threshold. Kidney Int 60:219-227

22. Koivisto VA, Stevens LK, Mattock M et al (1996) Cardiovascular disease and its risk factors in IDDM in Europe. EURODIAB IDDM Complications Study Group. Diabetes Care 19:689-697

23. Kearney EM, Mount JN, Watts GF, Slavin BM, Kind PR (1987) Simple immunoturbidimetric method for determining urinary albumin at low concentrations using Cobas-Bio centrifugal analyser. J Clin Pathol 40:465-468

24. Bucolo G, David H (1973) Quantitative determination of serum triglycerides by the use of enzymes. Clin Chem 19:476-482

25. Siedel J, Hagele EO, Ziegenhorn J, Wahlefeld AW (1983) Reagent for the enzymatic determination of serum total cholesterol with improved lipolytic efficiency. Clin Chem 29:1075-1080

26. Warnick GR, Albers JJ (1978) A comprehensive evaluation of the heparin-manganese precipitation procedure for estimating high density lipoprotein cholesterol. J Lipid Res 19:65-76

27. Friedewald WT, Levy RI, Fredrickson DS (1972) Estimation of the concentration of low-density lipoprotein cholesterol in plasma, without use of the preparative ultracentrifuge. Clin Chem 18:499 502

28. John WG, Gray MR, Bates DL, Beacham JL (1993) Enzyme immunoassay - a new technique for estimating hemoglobin A1c. Clin Chem 39:663-666

29. Ford I, Malik RA, Newrick PG et al (1992) Relationships between haemostatic factors and capillary morphology in human diabetic neuropathy. Thromb Haemost 68:628-633

30. Crook M (1993) The determination of plasma or serum sialic acid. Clin Biochem 26:31-38

31. Greaves M, Malia RG, Goodfellow K et al (1997) Fibrinogen and von Willebrand factor in IDDM: relationships to lipid vascular risk factors, blood pressure, glycaemic control and urinary albumin excretion rate: the EURODIAB IDDM Complications Study. Diabetologia 40:698-705

32. Aldington SJ, Kohner EM, Meuer S, Klein R, Sjolie AK (1995) Methodology for retinal photography and assessment of diabetic retinopathy: the EURODIAB IDDM complications study. Diabetologia 38:437-444

33. The Consensus Committee of the AAS and the AAN (1996) Consensus statement on the definition of orthostatic hypotension, pure autonomic failure, and multiple system atrophy. The Consensus Committee of the American Autonomic Society and the American Academy of Neurology. Neurology 46:1470

34. Tesfaye S, Chaturvedi N, Eaton SE et al (2005) Vascular risk factors and diabetic neuropathy. N Engl J Med 352:341-350

35. Prineas RJ, Crow RS, Blackburn H (1982) The Minnesota code manual of electrocardiographic findings. John Wright-PSG, Boston

36. Knuiman MW, Watts GF, Divitini ML (2004) Is sialic acid an independent risk factor for cardiovascular disease? A 17-year follow-up study in Busselton, Western Australia. Ann Epidemiol 14:627-632

37. Yokoyama H, Jensen JS, Myrup B et al (1996) Raised serum sialic acid concentration precedes onset of microalbuminuria in IDDM. A 10-year follow-up study. Diabetes Care 19:435-440

38. Danesh J, Lewington S, Thompson SG et al (2005) Plasma fibrinogen level and the risk of major cardiovascular diseases and nonvascular mortality: an individual participant meta-analysis. JAMA 294:1799-1809 
39. Danesh J, Collins R, Appleby P, Peto R (1998) Association of fibrinogen, C-reactive protein, albumin, or leukocyte count with coronary heart disease: meta-analyses of prospective studies. JAMA 279:1477-1482

40. Ernst E, Resch KL (1993) Fibrinogen as a cardiovascular risk factor: a meta-analysis and review of the literature. Ann Intern Med 118:956-963
41. Maresca G, Di Blasio A, Marchioli R, Di Minno G (1999) Measuring plasma fibrinogen to predict stroke and myocardial infarction: an update. Arterioscler Thromb Vasc Biol 19:1368-1377

42. Folsom AR, Wu KK, Rasmussen M et al (2000) Determinants of population changes in fibrinogen and factor VII over 6 years: the Atherosclerosis Risk in Communities (ARIC) Study. Arterioscler Thromb Vasc Biol 20:601-606 